medgen $2018 \cdot 30: 306-317$

https://doi.org/10.1007/s11825-018-0201-7

Online publiziert: 11 . Oktober 2018

(c) Der/die Autor(en) 2018

CrossMark

\author{
André Reis ${ }^{1}$ Frank M. Spinath ${ }^{2}$ \\ ${ }^{1}$ Humangenetisches Institut, Universitätsklinikum Erlangen, Friedrich-Alexander-Universität Erlangen- \\ Nürnberg (FAU), Erlangen, Deutschland \\ ${ }^{2}$ Fachbereich Psychologie, Universität des Saarlandes, Saarbrücken, Deutschland
}

\title{
Genetik der allgemeinen kognitiven Fähigkeit
}

ten Faktoren (• Abb. 1; vgl. [29]; für einen kompakten Überblick über die einschlägigsten strukturellen Ansätze s. [21]).

Darüber hinaus spielt die Intelligenz eine große Rolle in der Erklärung und Vorhersage individueller Unterschiede in zentralen Bereichen des gesellschaftlichen Lebens und Vorankommens, darunter Schul- und Bildungserfolg [39], Berufserfolg [41], sozioökonomischer Status [47] und Gesundheitsverhalten [5]. Die mit Intelligenz assoziierten Effektstärken sind dabei teils beträchtlich. So berichten Roth et al. in ihrer Metanalyse auf der Grundlage einer 105.185 Individuen umfassenden Gesamtstichprobe aus 240 unabhängigen Studien einen Zusammenhang zwischen Intelligenz und Schulnoten von $p=0,54$ (korrigierte Populationskorrelation), was Intelligenz zum mit Abstand stärksten Prädiktor von Schulerfolg macht [39]. Ein umfassender Überblick über weitere Befunde zur Intelligenz als Erfolgsfaktor findet sich bei Deary (2012; [10]).

\section{Wie wird Intelligenz gemessen?}

Es existieren zahlreiche psychometrische Tests zur Messung der Intelligenz, die hinsichtlich Aufgabenformat und erfassten Teilleistungen voneinander abweichen können. Zur Individualdiagnostik sind Testbatterien gebräuchlich (etwa der Intelligenz-Struktur-Test, I-S-T 2000 R; [1]), die Aufgaben zur Erfassung verschiedener Primärfaktoren der Intelligenz enthalten, darunter beispielsweise induktives Denken, verbales Verständnis, figurale Beziehungen und Umgang mit Zahlen. Empirisch zeigt sich mit hoher Konsistenz, dass die Leistungen in diesen verschiedenen Teilbereichen positive Interkorrelationen aufweisen, was als positive Mannigfaltigkeit bezeichnet wird und die Voraussetzung für die Aggregation derartiger Teilleistungen zu einem Gesamtwert darstellt [7]. Einschlägige Intelligenztestverfahren ermöglichen die Bestimmung eines solchen Gesamtwertes für jede getestete Person. Unter Heranziehung von Normwerten können diese dann in unmittelbar interpretierbare Standardwerte überführt werden, beispielsweise den Intelligenzquotienten (IQ; Mittelwert $M=100$, Standardabweichung $s=15$ ). Interindividuelle Differenzen in Intelligenzwerten weisen eine hohe zeitliche Stabilität auf, die ab dem jungen Erwachsenenalter Werte von $r=0,60$ und darüber annimmt [16].

\section{Erblichkeit von Intelligenz}

Verhaltensgenetische Studien haben in den vergangenen Jahrzehnten konsistent ergeben, dass genetische Einflüsse einen substanziellen Beitrag zur Erklärung individueller Unterschiede in der Intelligenz leisten [35], was im Einklang mit frühen Überblicksarbeiten auf der Grundlage von Zwillingsdaten sowie weiterer Verwandtschaftsgruppen steht [2]. Jüngere Überblicksartikel [3] zeigen zudem einen Zuwachs der Bedeutung genetischer Einflüsse über die Lebensspanne. Während im frühen Kindesalter vor allem Effekte der geteilten Umwelt (c2; Umwelteinflüsse, die zur Ähnlichkeit gemeinsam aufwachsender Individuen beitragen) für die Erklärung der Unterschiede in Intelligenzwerten verantwortlich sind, spielen diese bereits 


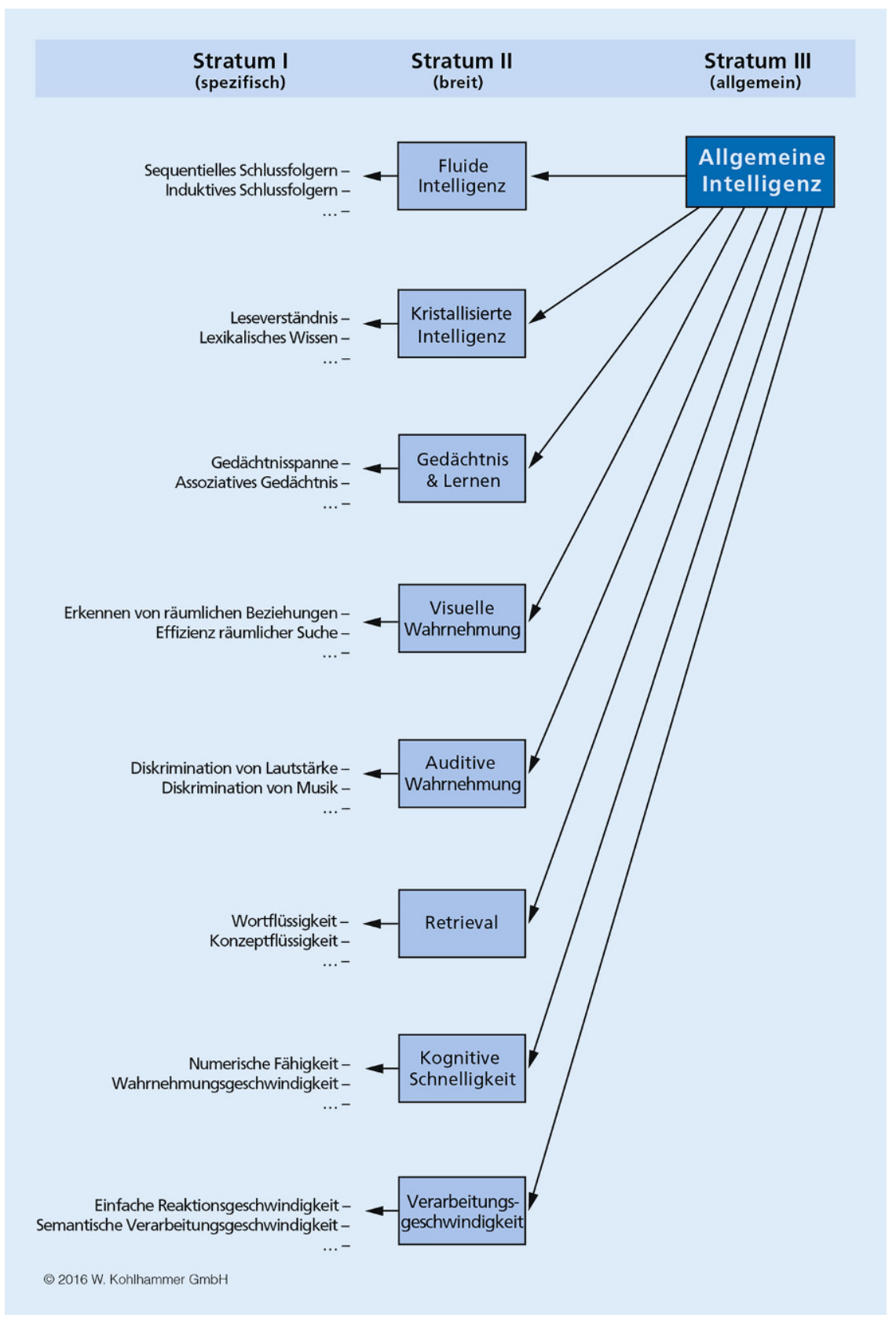

Abb. 1 А Auf der Re-Analyse von 460 Untersuchungen aus den Jahren 1930 bis 1985 beruhendes 3-Schichten-Modell (Three Stratum Model) der Intelligenz nach Carroll (1993; [7]).

(Abbildung aus Stemmler, Hagemann, Amelang und Spinath [46] mit freundlicher Genehmigung der W. Kohlhammer GmbH, @ 2016 W. Kohlhammer GmbH, 8. Auflage)

ab dem frühen Erwachsenenalter keine bedeutsame Rolle mehr. Der Abnahme der Bedeutung geteilter Umwelteinflüsse steht die Zunahme der Bedeutung genetischer Einflüsse gegenüber, die von etwas mehr als $20 \%$ der Intelligenzunterschiede im frühen Kindesalter über ca. $40-50 \%$ zum Schulanfang bis hin zu $60 \%$ und mehr im Erwachsenenalter erklären. Als eine mögliche Ursache
Korrelation (rGU; systematischer Zusammenhang zwischen Genotypen und bestimmten Umweltbedingungen) betrachtet. Neben der aktiven Form der rGU beschreiben Plomin, DeFries, \& Loehlin (1977) weiterhin die passive und die evokative Variante [33]. Passive rGU liegt beispielsweise vor, wenn Eltern Umwelten bereitstellen, die dem Genotyp des Kindes entgegenkommen/mit ihm assoziiert sind, während eine evokative rGU den Umstand widerspiegelt, dass vonseiten der Umwelt auf genetisch (mit)bedingtes Verhalten reagiert wird.

Demgegenüber beschreibt die Genotyp-Umwelt-Interaktion ( $\mathrm{GxU})$ die genetisch bedingte „Empfänglichkeit“ für Umwelteinflüsse. Als Scarr-Rowe-Hypothese bekannt wurde beispielsweise die Annahme, dass die Erblichkeit der Intelligenz am privilegierteren Ende der sozioökonomischen Verteilung höher ausfällt, während am unteren Rand der sozioökonomischen Verteilung geteilte Umwelteffekte bedeutsamer sind. Dies steht mit der Überlegung in Einklang, dass genetisches Potenzial sich unter günstigeren Umweltbedingungen stärker entfalten kann [51]. Belege für diese Annahme stammen derzeit vornehmlich aus US-amerikanischen Stichproben $[49,50]$.

\section{Erste genomweite Assoziations- studien}

Etwa 2005 begann mit der Etablierung genomweiter Assoziationsanalysen („genome-wide association study", GWAS) eine beispiellose Welle der Aufklärung genetischer Faktoren bei genetisch komplexen Erkrankungen und Merkmalen, die bis heute anhält. Bis Ende August 2018 enthielt der internationale Katalog aller publizierten GWAS, der beim European Bioinformatics Institute (EBI) geführt wird, insgesamt 3541 Publikationen und 69.969 unabhängige SNP-Merkmal-Assoziationen [26]. Es bestand die Hoffnung, mit dieser Technologie auch ein Werkzeug zur Aufklärung der genetischen Grundlagen der Intelligenz in Händen zu halten. Ein quantitatives Merkmal mit hoher Erblichkeit erschien ein ideales Anwendungsgebiet.

Umso größer war die Enttäuschung, als selbst für GWAS-Verhältnisse gro- 
Be und damit scheinbar gut gepowerte Studien ohne durchschlagenden Erfolg blieben. Als Beispiel möge eine große Metaanalyse des Cognitive Genomics Consortium (COGENT) an 35.298 gesunden Probanden europäischer Abstammung aus insgesamt 24 Einzelstudien dienen [48]. Diese generierte nur zwei genomweit signifikant assoziierte SNPLoci. Selbst die Zusammenfassung von verschiedenen Einzel-SNPs in einer genbasierten Analyse ergab nur 7 Genloci, die nach Bonferroni-Korrektur für multiple Testung noch signifikant waren. Ein weiterer wichtiger Parameter war ebenfalls nicht erfüllt: die erfolgreiche Replikation von Assoziationsbefunden. Die jeweiligen Assoziationsbefunde aus insgesamt 7 GWAS sowie die aus zahlreichen Kandidatengen-Studien replizierten weitgehend nicht, was letztlich die Möglichkeit eines falsch-positiven Befundes, trotz der Bonferroni-Korrektur, nicht überzeugend widerlegen konnte. Außerdem erklärten diese Befunde zusammen nur etwa $1 \%$ der Gesamtvarianz des Phänotyps. Entsprechend war die Effektstärke der einzelnen Loci äußerst gering, wodurch offenbar wurde, dass Intelligenz extrem heterogen ist und durch Tausende genetischer Faktoren mit jeweils sehr geringer Effektstärke beeinflusst ist. Deshalb wären extrem große Stichproben notwendig, um sie nachzuweisen.

\section{"Educational attainment" als Proxy-Phänotyp}

Eine interessante und wichtige Neuerung brachten Studien, die statt der aufwendig zu messenden kognitiven Funktion lediglich die abgeschlossenen Jahre schulischer/akademischer Bildung als Stellvertreter („proxy“) untersuchten. Dieser Parameter wird als Bildungsniveau oder „educational attainment“ (EA) bezeichnet. Verschiedene Studien haben gezeigt, dass er stark mit kognitiven Fähigkeiten korreliert (s.oben) [6]. Da dieser Parameter relativ leicht aus anamnestischen Angaben zu erheben ist, gelang es durch Zusammenführung zahlreicher Einzelstudien in einer Metaanalyse an 125.000 Individuen, drei genomweit signifikante SNPs zu identifizieren [37]. Die-

medgen 2018 · 30:306-317 https://doi.org/10.1007/s11825-018-0201-7

( ) Der/die Autor(en) 2018

\section{A. Reis · F. M. Spinath}

\section{Genetik der allgemeinen kognitiven Fähigkeit}

\section{Zusammenfassung}

Intelligenz ist eines der bestuntersuchten Konstrukte der empirischen Verhaltenswissenschaften und stellt eine allgemeine geistige Kapazität dar, die unter anderem die Fähigkeit zum schlussfolgernden Denken, zum Lösen neuartiger Probleme, zum abstrakten Denken sowie zum schnellen Lernen umfasst. Diese kognitiven Fähigkeiten spielen eine große Rolle in der Erklärung und Vorhersage individueller Unterschiede in zentralen Bereichen des gesellschaftlichen Lebens, wie Schul- und Bildungserfolg, Berufserfolg, sozioökonomischer Status und Gesundheitsverhalten. Verhaltensgenetische Studien zeigen konsistent, dass genetische Einflüsse einen substanziellen Beitrag zur Erklärung individueller Unterschiede leisten, die über $60 \%$ der Intelligenzunterschiede im Erwachsenenalter erklären. In den letzten Jahren konnten in großen genomweiten Assoziationsstudien mit häufigen genetischen Varianten Hunderte mit Intelligenz assoziierte Loci identifiziert werden sowie über 1300 assoziierte Gene mit differentieller Expression überwiegend im Gehirn. Mehrere
Signalwege waren angereichert, vor allen für Neurogenese, Regulation der Entwicklung des Nervensystems sowie der synaptischen Struktur und Aktivität. Die Mehrzahl der assoziierten Loci betraf regulatorische Regionen und interessanterweise lag die Hälfte intronisch. Von den über 1300 Genen überlappen nur 9,2\% mit solchen, die mit monogenen neurokognitiven Störungen assoziiert sind. Insgesamt bestätigen die Befunde ein polygenes Modell Tausender additiver Faktoren, wobei die einzelnen Loci eine sehr geringe Effektstärke aufweisen. Insgesamt erklären die jetzigen Befunde ca. $10 \%$ der Gesamtvarianz des Merkmals. Diese Ergebnisse sind ein wichtiger Ausgangspunkt für zukünftige Forschung sowohl in der Genetik als auch den Verhaltenswissenschaften.

\section{Schlüsselwörter}

Intelligenz · Erblichkeit · Genomweite Assoziationsstudie $\cdot$ Polygenic score . Prädiktion

\section{Genetics of general cognitive function}

\section{Abstract}

Intelligence is one of the best studied constructs of empirical behavioral sciences and represents a general cognitive capacity, which includes - among others - the ability for conceptual thinking, solving challenging problems, abstract thinking, and rapid learning. These cognitive functions play an enormous role in the explanation and prediction of individual differences in central areas of societal life, e. g., schooling and educational success, professional success, socioeconomic status, and health-related behavior. Behavioral genetic studies have consistently shown that genetic influences make a substantial contribution to defining individual differences, that explain more than $60 \%$ of variations in intelligence in adults. Over the last few years, in large genome-wide association studies using frequent genetic variants, hundreds of loci associated with intelligence were identified, in addition to more than 1300 associated genes, which were differentially expressed in the brain. Several pathways were overrepresented, mainly those for neurogenesis, the regulation of nervous system development, and the regulation of synapse structure and activity. Most associated loci were located in regulatory regions and, interestingly, half of them in introns. Of the more than 1300 associated genes, only $9.2 \%$ overlapped with those associated with monogenic cognitive defects. Overall, the findings confirm a polygenic model of thousands of additive factors, in which individual loci have a very small effect. Collectively, the current results explain up to $10 \%$ of the overall variance in cognitive function. These results are an important starting point for future research, not only in genetics but also in the behavioral sciences.

\section{Keywords}

Intelligence · Heritability · Genome-wide association study · Polygenic score - Prediction 


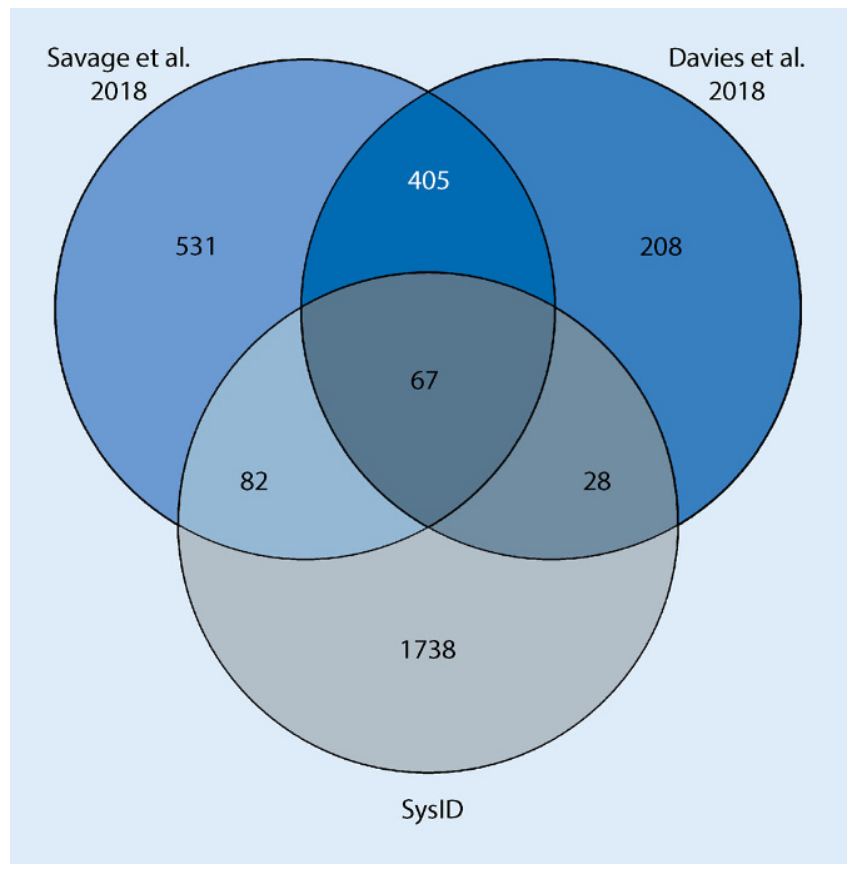

Abb. 2 \ Vergleich derÜberlappung der mit Intelligenzassoziierten Gene aus den Studien von Savage et al. [40] und Davies et al. [8] mit den mit kognitiven Störungen assoziierten Genen aus der SysID-Datenbank (Stand Juni 2018; [22])

se konnten erstmals in unabhängigen Kohorten repliziert werden [36]. Die Effektstärken waren auch hier sehr klein, der stärkste Effekt eines SNPs (rs9320913) entsprach lediglich $0,02 \%$ der Gesamtvarianz in der Replikationskohorte.

In Anbetracht dieser minimalen Effektstärken einzelner SNPs wurde die Analyse auf die Gesamtheit der assoziierten SNPs verlagert, unabhängig davon, ob sie die strengen statischen Schwellenwerte der Signifikanz erreichten, die sonst bei GWAS angewandt werden. Dieser als „polygenic score“ (PGS), „polygenic risk score", "genetic risk score" oder "genome-wide polygenic score" bezeichneter Gesamtwert stellt die gewichtete Summe aller (assoziierten) SNPs dar und soll als bester Prädiktor für das Merkmal dienen. (Für einen aktuellen Überblick zur Risikostratifikation mittels PGS bei verschiedenen komplexen Erkrankungen s. [20]) Dieser PGS konnte 2\% der Gesamtvarianz in einer Replikationsstudie erklären, also 100-fach mehr Varianz als der beste Einzel-SNP [37].

Ermutigt durch diesen Erfolg wurde 2016 eine zweite Metaanalyse zu EA an nunmehr 294.000 Individuen durchgeführt [31]. Bei dieser Stichprobengröße waren 74Locigenomweit signifikant, und die Gesamtvarianz für EA, die der PGS erklären konnte, erreichte $3 \%$. Interessanterweise erklärte der PGS sogar 4\% der Varianz für Intelligenz, also ein höherer Wert als der eigentliche Zielphänotyp der Studie.

\section{Mega-GWAS}

Den Durchbruch brachten aber große Metaanalysen für den Phänotyp Intelligenz. Der Phänotyp war in den einzelnen Studien zwar mit unterschiedlichen, aber letztlich vergleichbaren Methoden gemessen worden, sodass sie kombiniert werden konnten. Die Arbeit von Sniekers et al. erschien 2017 und fand bereits unter 78.308 Individuen 336 assoziierte SNPs und 52 assoziierte Gene [43]. Eine noch größere Studie von Savage et al. von Juni 2018 [40] aus derselben Arbeitsgruppe von Danielle Posthuma aus Amsterdam vervierfachte praktisch die Stichprobengröße auf fast 280.000 Individuen aus 16 unabhängigen Kohorten inklusive der großen Studien der UK Biobank und der o.g. COGENT-Studie, aber auch teilweise der schon bei Sniekers et al. verwendeten Kohorten [43]. Insgesamt waren
531 unabhängige SNPs genomweit signifikant, die wiederum 246 unabhängigen Loci zugeordnet werden konnten. Wie auch bei anderen komplexen Merkmalen stieg die Zahl der signifikant assoziierten Gene nicht linear, sondern wurde sogar verzwanzigfacht auf nunmehr 1041. Dies war möglich durch die Zusammenlegung aller assoziierter SNPs einzelner Gene, auch wenn die jeweiligen SNPs nicht die Signifikanzschwelle für die genomweite Assoziation überschritten. Eine Replikation der Befunde gelang in einem Teil der UK Biobank $(n=188.000)$, deren Individuen keine formelle Intelligenztestung erfahren hatten, aber für die der hochkorrelierte Phänotyp EA verfügbar war. Insgesamt zeigten $94 \%$ der SNPs die gleiche Richtung der Assoziation, und 51 Loci zeigten Evidenz einer Replikation. Insgesamt erklärten die jetzt identifizierten SNPs 5,4\% der Gesamtvarianz des Merkmals Intelligenz [40].

Einen ähnlichen Ansatz wählten Davies und Mitarbeiter (2018), die sogar 300.486 Individuen aus 57 populationsbasierten Studien analysierten [8]. Diese Studie greift allerdings auf dieselben UK Biobank-Proben zurück wie die Gruppe von Posthuma, sodass eine erhebliche Überlappung der Kohorten besteht. Die Studien unterscheiden sich allerdings in technischer Hinsicht insbesondere bezüglich der Qualitätssicherung der Genotypen aus den verschiedenen Ursprungsstudien. Das erklärt vielleicht, dass Davies et al. nur 148 unabhängige Loci und 709 mit Intelligenz assoziierte Gene fanden. Auch wenn die Ergebnisse beider Studien erheblich überlappen (- Abb. 2) bleibt bemerkenswert, dass durch die Hinzunahme divergenter weiterer Kohorten jeweils andere Loci und Gene statistisch signifikant werden. Dies deutet darauf hin, dass das Merkmal außerordentlich heterogen ist und die Ergebnisse zahlreicher Loci knapp unterhalb der statistischen Signifikanzschwelle liegen dürften.

Die größte GWAS bisher wurde schließlich wiederum zum (Proxy-) Phänotyp EA im Juli 2018 veröffentlicht. Eine Metaanalyse von 71 Kohortenstudien erweitert die Stichprobengröße gegenüber früheren Studien auf insgesamt 1,1 Mio. Menschen. Lee und Kollegen 
Tab. 1 Alphabetische Liste der 177 Gene aus dem Vergleich der mit Intelligenz assoziierten Gene aus den Studien von Savage et al. [40] und Davies et al. [8] mit den mit kognitiven Störungen assoziierten Genen aus der SysID-Datenbank (Stand Juni 2018; [22]) siehe Abb. 2

Gene für kognitive Fähigkeiten und für kognitive Störungen

\begin{tabular}{|c|c|c|c|}
\hline$A C O 2$ & DLGAP1 & $M A D D$ & SALL1 \\
\hline AFF3 & DLL4 & MAN2B1 & SARS \\
\hline ALMS1 & DNM3 & MCEE & SATB2 \\
\hline$A M T$ & DOCK3 & $M E C R$ & $S C N 2 A$ \\
\hline ANK2 & $D P Y D$ & MEF2C & SEMA3E \\
\hline$A P 3 B 2$ & $E F C A B 5$ & MICU1 & SHANK3 \\
\hline APOPT1 & EFTUD2 & MRPL10 & SIX3 \\
\hline ARFGEF2 & EP300 & MSMO1 & $S L C 25 A 20$ \\
\hline ARHGAP15 & EPB41L1 & MY018B & SLC39A8 \\
\hline ARID1B & $E R C C 8$ & $N A G A$ & SLC6A9 \\
\hline ARID2 & FBXL4 & NDUFA2 & SMARCC2 \\
\hline$A R L 13 B$ & FBXO31 & NDUFAF2 & SMYD5 \\
\hline ATF7IP & FOXP1 & NDUFAF3 & $S O B P$ \\
\hline$A T P 2 A 1$ & FRRS1L & NDUFS3 & SOX11 \\
\hline ATPAF2 & FUT8 & NEU1 & SPAST \\
\hline AUTS2 & GAD1 & $N F I X$ & ST3GAL3 \\
\hline$B C L 11 A$ & GATAD2B & $N R 2 F 1$ & STAG1 \\
\hline BRWD1 & $G C D H$ & NR4A2 & STAMBP \\
\hline$B S N$ & GFAP & NRXN1 & suox \\
\hline BTN1A1 & GLYCTK & NTNG2 & SYT1 \\
\hline BTN2A2 & GMPPA & ORC1 & $S Z T 2$ \\
\hline BTN3A2 & GMPPB & $P C C B$ & $T A T$ \\
\hline C12orf65 & GNAI2 & PCDHA13 & TBR1 \\
\hline CACNA1D & GNB5 & PDE4D & TBRG1 \\
\hline CACNA1E & GPM6A & PEX16 & TCF20 \\
\hline CACNA2D2 & GPR52 & PHF2 & TCF4 \\
\hline CACNA2D3 & GRIA4 & PIGW & THRB \\
\hline CAMTA1 & GRID2 & PIK3R2 & $T L K 2$ \\
\hline CASC5 & GRIN2A & PITX3 & TOE1 \\
\hline$C D C 42$ & HAX1 & PLCB1 & TRAIP \\
\hline CEP57 & HIST1H4B & PLK2 & TREX1 \\
\hline CHP1 & HIST1H4C & POU3F2 & TRIM37 \\
\hline CLN3 & INO80 & PPRC1 & TROVE2 \\
\hline CLP1 & IVD & PRKD1 & TSFM \\
\hline CNOT4 & KALRN & PRMT7 & TSHZ3 \\
\hline CNTNAP4 & KANSL 1 & PTCH1 & TUBAL3 \\
\hline COG5 & KCNB1 & PTK7 & UBA7 \\
\hline$C P E$ & KCNJ6 & PTPRT & VAC14 \\
\hline CTNNA2 & KIF16B & QARS & WWP2 \\
\hline CTNNB1 & $K M T 2 D$ & QRICH1 & $\mathrm{ZC} 3 \mathrm{H} 4$ \\
\hline CTNND1 & KMT2E & $R A B 11 A$ & ZNF335 \\
\hline CWF19L1 & LARGE & RAl1 & ZSWIM6 \\
\hline$D A G 1$ & LARP7 & RBFOX1 & \\
\hline DARS2 & $L R P 1 B$ & $R C B T B 1$ & \\
\hline$D C C$ & LRPPRC & RFT1 & \\
\hline
\end{tabular}

(2018; [25]) fanden in dieser auch als EDU3 bezeichneten Studie insgesamt 1271 unabhängige SNPs für EA, die genomweit signifikant waren. Insgesamt über 1800 Gene waren assoziiert, etwa 10-mal so viele wie in der Studie zuvor zu EA. Die größere Stichprobe, aber auch Innovationen in der Datenanalyse führten dazu, dass die daraus generierten PGS in Replikationskohorten bis zu 9,7\% der Varianz für Intelligenz erklären, der höchste bisher erzielte Wert. Die durch die PGS erklärte Varianz für EA beträgt sogar $12 \%$.

Insgesamt konvergieren die Ergebnisse der verschiedenen Studien, wenngleich Unterschiede in der Größe und Zusammensetzung der Einzelkohorten sowie methodische Aspekte eine Rolle spielen.

\section{Genetische und molekulare Architektur}

Mit diesen Studien ist das Merkmal Intelligenz nun endgültig im Feld der genetisch komplexen Merkmale angekommen. Die Vergrößerung der Stichproben und Verbesserung der Methoden haben den Durchbruch ermöglicht und über tausend signifikante Assoziationsbefunde offenbart. Trotz extrem geringer Effektstärken einzelner SNPS konnte ein Teil bereits erfolgreich repliziert werden. Es zeichnet sich ein stark polygenes Merkmal ab, mit Tausenden von additiven Einzelfaktoren mit jeweils extrem geringer Effektstärke. Die Architektur des Merkmals ist ähnlich heterogen wie das Merkmal Körperhöhe, bei dem in ähnlich großen GWAS mit 3290 unabhängigen SNPs 24,6\% der phänotypischen Varianz erklärbar war [52].

Mit den Ergebnissen der jüngsten Studien aus 2018 von Davies et al. und Savage et al. $[8,40]$ ist es erstmals möglich, Einblicke nicht nur in die genetische, sondern auch in die molekulare Architektur der kognitiven Leistungsfähigkeit zu erhalten. Die Autoren beider Studien untersuchten die funktionellen Kategorien der assoziierten SNPs und die Expression der assoziierten Gene sowie deren Signalwege. Bemerkenswert sind einige Unterschiede in der genetischen Architektur im Vergleich zu anderen komplexen Merkmalen. So lagen $51 \%$ der SNPs 
für Intelligenz intronisch, $27 \%$ intergenisch und 1,5\% exonisch [40]. Savage et al. fanden auch 89 nicht synonyme exonische Varianten. Hingegen lagen bei einer gemeinsamen Analyse verschiedener chronisch entzündlicher Erkrankungen beispielsweise $86 \%$ der assoziierten SNPs zwar innerhalb von $10 \mathrm{~kb}$ eines benachbarten Gens, aber intergenisch, und $6,5 \%$ lagen exonisch [12].

Erwartungsgemäß enthalten die mit Intelligenz assoziierten SNP-Locihöchstwahrscheinlich regulatorische Elemente, denn sie liegen in evolutionär konservierten Regionen und überlappen mit Markern offenen Chromatins, bekannten regulatorischen Elementen und eQTLLoci. Ebenfalls nicht unerwartet ist das Expressionsmuster der assoziierten Gene. So waren die Gene statistisch signifikant im Gehirn und der Schilddrüse differentiell exprimiert. Auch verschiedene bereits mit kognitiven Fähigkeiten assoziierte Gehirnareale zeigten eine signifikante Anreicherung in der Expression, darunter diverse kortikale Regionen, Amygdala, Hippocampus, Nucleus caudatus und Putamen [8]. Die Zellen mit der höchsten Expression assoziierter Gene waren mittelgroße Projektionsneuronen des Striatum sowie kortikale und hippocampale Pyramidalneuronen [40].

Eine Analyse der Signalwege und biologischen Funktionen der assoziierten Gene zeigte eine signifikante Anreicherung für Neurogenese, also den Prozess der Bildung reifer Neuronen aus Stammzellen. Weitere assoziierte Prozesse waren die Regulation der Struktur und Prozesse der Synapse sowie der Regulation der Entwicklung des Nervensystems und der Regulation der Zellentwicklung allgemein [40].

Die funktionelle Annotation der in EDU3 mit EA-assoziierten Gene bestätigten frühere Befunde und erweiterten sie um zwei Punkte [25]. Die Expression der Gene erstreckte sich nicht mehr bevorzugt auf die pränatale Phase, sondern auch auf die postnatale Entwicklung. AuBerdem wurden insbesondere Gen-Netzwerke identifiziert, die an der Kommunikation zwischen Neuronen beteiligt sind. Bemerkenswert war auch das praktische Fehlen von Gen-Sets, die in Glia-Zellen differentiell exprimiert sind, obwohl diese Zellen etwa die Hälfte des zentralen Nervensystems ausmachen. Das legt nahe, dass die Geschwindigkeit der Neurotransmission keine größere Rolle für die Unterschiede in der Kognition spielt [25].

\section{Vergleich der Gene für kognitive Fähigkeiten und für kognitive Störungen}

Eine naheliegende Frage ist, ob dieselben Gene, die wenn mutiert neurokognitive Erkrankungen (der Schwerpunkt dieses Heftes) verursachen, auch mit Intelligenz assoziiert sind. Für neurokognitive Störungen sind nach mehr als drei Jahrzehnten intensiver Forschung inzwischen 1915 beteiligte Gene identifiziert (SysID-Datenbank, Stand Juni 2018; [22]), sodass nunmehr ein Vergleich möglich und sinnvoll erscheint. Die beiden größten Studien zur Intelligenz aus 2018, von Savage et al. und Davies etal. [8,40], haben insgesamt 1321 assoziierte Gene gefunden, wobei etwa ein Drittel (472) überlappt (• Abb. 2). Das Venn-Diagramm zeigt, dass immerhin 177 Gene $(9,2 \%)$ der mit neurokognitiven Störungen assoziierten Gene auch mit Intelligenz assoziiert sind (• Abb. 2; - Tab. 1). Hinsichtlich der Genetik wissen wir aktuell offenbar mehr über Wege, wie neurokognitive Funktionen gestört werden können, als über die Prozesse, die an der Modulierung von Fähigkeiten beteiligt sind.

Die überlappenden Gene sind signifikant angereichert für dieselben GeneOntology(GO)-Terms wie die für Intelligenz allgemein. Über die Hälfte (100; $57 \%$ ) war mit einem autosomal rezessiven Erbgang assoziiert, 75 mit einem dominanten und 2 mit beiden Erbgängen. Bei den dominanten zeigten 51 (68\%) Zeichen der Haploinsuffizienz (pLi Score $>0,9$ ). Möglicherweise wirken sich einige rezessive Gene doch im heterozygoten Zustand quantitativ aus. Es ist ebenfalls naheliegend, dass haploinsuffiziente Gene über regulatorische Effekte ebenfalls quantitativ und damit in ihrer Funktion modulierbar sind, da hier die Dosis besonders kritisch ist.

\section{Bezug zu psychiatrischen Erkrankungen und anderen Merkmalen}

Eine Assoziation von Intelligenz mit neuropsychiatrischen Erkrankungen wie Schizophrenie und manisch-depressiver Erkrankung war bereits zuvor bekannt. Insgesamt zeigen die genetischen Faktoren vieler dieser Erkrankungen eine starke Überlappung [13]. Zudem ist Intelligenz bereits früher mit verschiedenen anthropometrischen und medizinischen Merkmalen assoziiert worden. Diese Assoziationen sind nun auch auf SNPEbene durch den Abgleich mit publizierten GWAS bestätigt [8] bzw. mittels „Mendelian randomization“ untersucht worden. Die Mendel'sche Randomisierung [40] bezeichnet eine neuartige biostatistische Methode zur Bestimmung des Einflusses von robust assoziierten Genvarianten auf Merkmale. Dabei zielt sie auf einen unverzerrten Nachweis von kausalen Effekten und, wenn möglich, der Schätzung der Effektstärke. Hierbei fand sich eine große Übereinstimmung der Studien, wobei sich die stärkste positive Assoziation erwartungsgemäß mit EA fand. Außerdem für ehemaliges Rauchen, Autismus und intrakranielles Volumen sowie Langlebigkeit und das Tragen von Brillen oder Kontaktlinsen. Eine negative Korrelation, also ein protektiver Effekt der überlappenden genetischen Prädisposition zu einem hohen IQ, fand sich für Alzheimer, depressive Symptome, Schizophrenie, ADHS, Bluthochdruck, Lungenkrebs sowie Gewicht und Body-Mass-Index [40]. Dies deutet auf pleiotrope Faktoren hin, die für verschiedene Merkmale und Erkrankungen überlappen.

Interessant ist auch die Analyse der Genetik der Subphänotypen, die zur allgemeinen kognitiven Fähigkeit "g“ beitragen (• Abb. 1). Die bisher größte Studie hierzu analysierte eine sowohl phänotypisch als auch genotypisch einheitlich charakterisierte Kohorte von 112.151 Probanden aus der UK Biobank [9]. Dabei zeigten insbesondere die Subphänotypen „sprachlogisches Denken und Zahlenverständnis“ (31\%) und „EA“ (21\%) eine hohe SNP-basierte Heritabilität, während „Reaktionszeit“ 

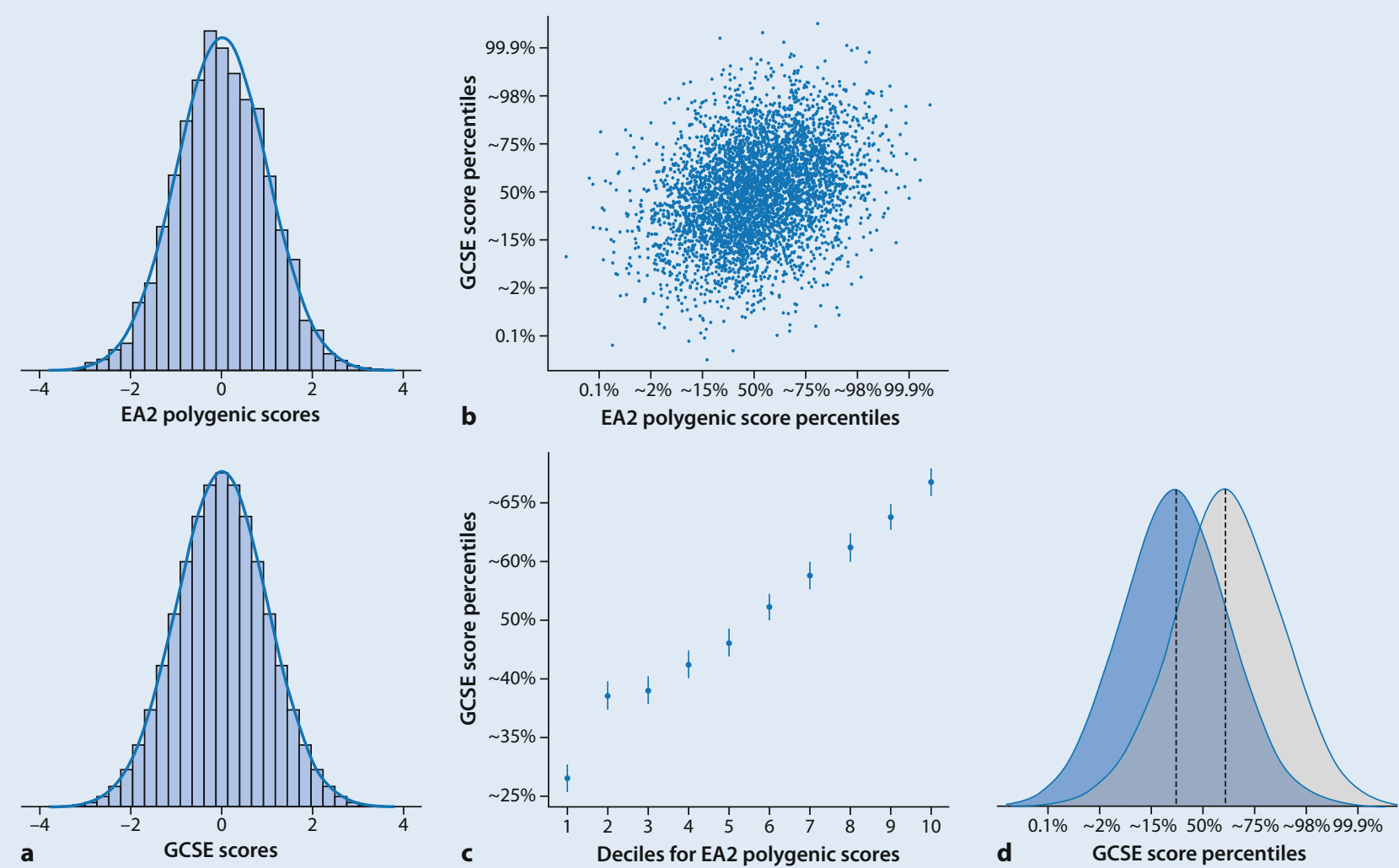

Abb. 3 ॥ Prädiktion kognitiver Fähigkeiten mithilfe von „polygenic score“.

a „Educational attainment polygenic score" (EA2) als Proxy-Phänotyp für kognitive Fähigkeiten und der Score im schulischen General-Certificate-of-Secondary-Education(GCSE)-Test sind normalverteilt. $\mathbf{b}$ Einzelwertkorrelation zwischen beiden Scores. c Die EA2-Dezile korrelieren mit den GCSE-Perzentilen. $\mathbf{d}$ Die obersten und untersten EA2-Dezile zeigen eine 61 \%ige Überlappung in den GCSE-Scores. Daten aus England aus der Selzam et al. Studie [42], für Details s. Text. Abbildung aus Plomin und von Stumm [34] mit freundlicher Genehmigung von Springer Nature

(11\%) und „Gedächtnis“ (8\%) eine deutlich geringere SNP-basierte Heritabilität zeigten. Dennoch waren erwartungsgemäß die Subphänotypen stark genetisch korreliert, so z. B. „sprachlogisches Denken und Zahlenverständnis“ und „EA“ mit einer Korrelation von $g=0,73$. Diese Studie zeigt auch, dass neben nicht genetischen Faktoren auch andere genetische Faktoren, die nicht mit kognitiven Fähigkeiten, sondern etwa mit Persönlichkeitsmerkmalen assoziiert sind, signifikant zur Variabilität des Merkmals EA beitragen.

\section{Werden Gentests zur Vorhersage von Intelligenz herangezogen werden können?}

Aktuell lassen sich ca. 11-13\% der Gesamtvarianz des EA und 7-10 \% der Intelligenz mithilfe von PGS vorhersagen [25].
Es ist absehbar, dass dieser Wert in den nächsten Jahren dank immer größerer GWAS und Verbesserungen in den statistischen Verfahren weiter ansteigen wird. Die theoretische Obergrenze der mit häufigen SNPs erklärbaren Varianz wurde auf etwa $25 \%$ geschätzt. Durch Berücksichtigung auch seltener Allele könnte diese Obergrenze allerdings sogar auf $50 \%$ ansteigen [19]. Es ist bereits jetzt erkennbar, dass versucht werden wird, diese PGS auch prognostisch, also vorhersagend, einzusetzen.

Hier gilt es jedoch zur Vorsicht zu mahnen. Ein PGS erlaubt lediglich eine probabilistische Aussage. So korreliert der Wert in einer untersuchten Gruppe bedeutsam mit der gemessenen Intelligenz, aber im Einzelfall kann ein gemessener Wert stark vom vorhergesagten Wert abweichen, weswegen PGS als prognostischer Marker für Individuen unge- eignet sind. Als Beispiel mag die Studie von Selzam et al. [42] dienen, die den PGS der Okbay et al. Studie [31] zum korrelierten (Proxy-)Phänotyp der „educational years" auf eine Kohorte von 5800 Schülern im Alter von 16 Jahren aus dem Vereinigten Königreich und deren Ergebnisse in einem nationalen Schulexamen (General Certificate of Secondary Education, GCSE) untersuchte. Das Examensergebnis ist normalverteilt, und der Wert wird als "educational achievement score" (EAS) bezeichnet (- Abb. 3a). Aus Zwillingsstudien war bekannt, dass die Erblichkeit dieses Scores bei $60 \%$ liegt [18]. Wie zu erwarten, war der PGS ebenso normalverteilt (• Abb. 3a).

Wenn die Kohorte nach ihren PGS in Dezile eingeteilt wird, dann differieren diese in ihren EAS entsprechend. Gemäß der Gesamtkorrelation lagen die Mittelwerte der untersten PGS-Dezile bei der 
28. Perzentile des EAS und die der obersten PGS-Dezile bei der 68. Perzentile des EAS (• Abb. 3c). Innerhalb einer PGSDezile gab es jedoch eine breite Varianz der EAS, die Überlappung der Verteilung des EAS beider Dezile betrug $61 \%$ (- Abb. 3d). Entsprechend lagen die Einzelwerte des EAS innerhalb einer PGSDezile weit auseinander: so hatte das Individuum mit dem zweithöchsten PGS nur einen EAS knapp über dem Mittelwert der Gesamtkohorte, ein Individuum mit dem achtniedrigsten PGS-Wert hatte einen EAS oberhalb der 75. Perzentile (• Abb. 3b). Während die Korrelation also auf die Gesamtkohorte valide ist, ist die Vorhersagegenauigkeit für den Einzelnen sehr gering.

Allerdings werden an den Rändern der Verteilung, also z. B. in den untersten oder obersten Perzentilen, bessere Vorhersagen möglich, sofern modifizierende Effekte eines (niedrigen) sozioökonomischen Status ausgeschlossen sind. In diesem Zusammenhang ist eine gerade erschienene Arbeit der DDD Studie relevant [30]. Die Autoren zeigen, dass ein PGS zum Risiko für kognitive Störungen beiträgt und postulieren, dass ein Teil der NDD-Patienten, insbesondere die mit einem leichteren Schweregrad, keine monogene Ursache haben sondern nur den unteren Bereich der polygenen Normalverteilung darstellen. In dieser Studie war der PGS entsprechend vergesellschaftet mit niedrigerer Intelligenz und Bildungserfolg und mit einem erhöhten Risiko für Schizophrenie. Langfristig ist ein PGS-basierter Prädiktionswert zu erwarten, der beispielsweise dem der koronaren Herzerkrankung entspricht, wobei die obersten $8 \%$ der Bevölkerung eine Risikoerhöhung $(\mathrm{OR} \geq 3)$ vergleichbar mit dem einer monogenen familiären Hypercholesterinämie aufweisen [20].

\section{Genetik der Umwelt}

Ein wichtiger Aspekt in der Diskussion um Genetik und Umwelt („nature and nurture") ist die Beobachtung von Kong et al. [24], dass die elterlichen Genotypen selbst dann einen Effekt auf das EA der Kinder haben, wenn sie nicht vererbt werden. In einer Nachanalyse einer isländischen Teilkohorte der EA-Studie von Ok- bay et al. [31] konnten sie zeigen, dass der PGS der nicht vererbten (nicht transmittierten) Allele der Eltern etwa 30\% der Varianz erklärte, die für den PGS der vererbten (transmittierten) Allele errechnet wurde. Die nicht transmittierten Allele wirken nicht direkt auf die Kinder, sondern auf die Eltern. Diese schaffen in der Folge eine entsprechende Umwelt („nurture"), die im Sinne einer passiven Genotyp-Umwelt-Korrelation auf die Kinder wirkt. Genetische- und Umweltmechanismen auf das Merkmal sind also eng verwoben. Dieser Wert ist für EA mit $30 \%$ deutlich größer als etwa für die Körperlänge, für die der analoge Wert nur $6 \%$ beträgt. Dieser Aspekt, der als „genetic nurture effect" apostrophiert wurde [23], ist in GWAS bisher nicht berücksichtigt worden und trägt möglicherweise dazu bei, dass die o.g. Vorhersageanteile der PGS überschätzt sind. Die zukünftige Untersuchung nicht transmittierter Allele verspricht aber neue Wege in der Analyse der beteiligten Faktoren und Signalwege und unterstreicht die Bedeutung genetischer Strategien (Trio vs. Einzelfall) in der Erforschung kognitiver Merkmale.

\section{Zukünftige Forschung}

Die weitere Entwicklung der Forschung kann anhand von Beispielen aus dem Bereich komplexer Erkrankungen teilweise vorausgesagt werden. Die untersuchten Kohorten werden immer größer werden, wie zuletzt bei Lee et al. [25] (1,1 Mio. Individuen), insbesondere für leicht zu erhebende Proxy-Phänotypen wie EA. Auch große populationsbasierte Kohorten wie die UK Biobank, die bereits jetzt einen erheblichen Beitrag geleistet haben, werden weiter anwachsen. Die Power wird dadurch, wie dies bereits in den aktuellen Studien deutlich wird, überproportional anwachsen und die Zahl der Assoziationsbefunde bzw. deren statistische Sicherheit entsprechend steigen.

Bisher wurden mithilfe der relativ kostengünstigen Arrays allerdings meist nur häufige SNPs mit einer Frequenz des seltenen Allels $>1 \%$ untersucht. Mit dem Übergang zu den zwar teureren, aber vollständigeren sequenzbasierten Genotypisierungstechniken werden auch sel- tenere Varianten in den Fokus geraten. Auch hier ist der Vergleich zum polygenen Merkmal Körperhöhe aufschlussreich. In einer groß angelegten Studie zu seltenen Allelen fanden die Autoren 83 mit Körperhöhe assoziierte kodierende Varianten mit einer Allelfrequenz von $0,1-4,8 \%$ und Effektstärken von bis zu $2 \mathrm{~cm}$ pro Allel, etwa 10 -mal so viel wie der durchschnittliche Effekt häufiger Allele [27].

Bisherige GWAS basieren auf einem einfachen additiv-genetischen Modell ohne Berücksichtigung möglicher interaktiver Effekte (Epistase). Grundsätzlich sind aber auch multiplikative oder vollständig andere kombinatorische Effekte zwischen einzelnen Loci zu erwarten. Letztere sind jedoch aufgrund der exponentiell wachsenden Zahl von Tests, für die statistisch zu korrigieren wäre, praktisch nur dann nachweisbar, wenn zuvor pathophysiologische Überlegungen eine reduzierte Zahl von Assoziationen begründen. Entsprechend wenige Beispiele für solche Effekte sind publiziert (z. B. für Psoriasis [14]). Für einen genomweiten Ansatz hat praktisch keine Studie die notwendige Power.

Auch wurden bisher noch keine Kopienzahlvarianten („copy number variants“, CNVs) systematisch untersucht. Aus der Forschung an kognitiven Störungen und neuropsychiatrischen Erkrankungen ist die enorme Bedeutung der CNVs und anderer struktureller Varianten jedoch bestens bekannt. Insbesondere Mikrodeletionssyndrome könnten einen erheblichen Effekt auf kognitive Fähigkeiten haben, der größer sein könnte als die aktuellen PGS. Sie stellen somit seltene Allele mit großer Effektstärke dar. Auch bei der Körperhöhe spielen seltene CNVs mit großer Effektstärke eine signifikante Rolle [53]. Mit den bisherigen arraybasierten Techniken sind CNVs in großen Kohorten kaum zuverlässig zu bestimmen. Dies sollte aber mit sequenzbasierten Techniken wie "whole genome sequencing" sehr viel besser möglich sein.

Zukünftige Studien müssen auch den Beitrag des X-Chromosoms berücksichtigen. Derzeitige GWAS-Studiendesigns lassen dieses Chromosom weitgehend aus. Die EDU3-Studie aus 2018 [25] 
hat zwar dieses Chromosom erstmals an einem Teil des Datensatzes getrennt analysiert. Es fanden sich lediglich 10 assoziierte Loci und der Beitrag zur Varianz wurde auf $0,3 \%$ geschätzt, weniger also als ein Autosom vergleichbarer Größe. Allerdings bleiben angesichts noch offener methodischer Fragen und in Hinblick auf die relativ kleine Untergruppe viele Fragen offen. In Anbetracht der Bedeutung X-chromosomaler Gene für neurokognitive Erkrankungen ist zu erwarten, dass auch hier mit Intelligenz assoziierte Gene zu finden sind.

Bisherige Studien haben sich weitgehend auf Europäer konzentriert. Deshalb sind Studien auch an anderen Populationen notwendig, auch wenn grundsätzlich keine anderen Gene oder beteiligte Signalwege als bei Europäern zu erwarten sind, teilweise aber andere Allele. So verwundert es nicht, dass der prädiktive Wert der in der EDU3-Studie ermittelten PGS in einer Untergruppe afroamerikanischer Personen einen signifikant niedrigeren Vorhersagewert aufwies als das für Menschen europäischer Abstammung der Fall war [25]. Diese Studien werden auf jeden Fall die Gesamtpower erhöhen und so auch zur Replikation und Verfeinerung der Assoziationsbefunde beitragen.

Es ist möglich, dass einige der jetzt benannten Gene falsch-positive Befunde darstellen, da die genbasierte GWASMethodik keine Assoziationen außerhalb der Gene berücksichtigt. Es ist somit nicht auszuschließen, dass die assoziieren SNPs in einem Gen eigentlich ein anderes (benachbartes) Gen regulieren. Letztlich müssen funktionelle Studien helfen, die molekularen Mechanismen aufzuklären. Dies wird aber wegen der geringen Effektstärke der einzelnen Loci eine besondere Herausforderung sein. Hierfür könnten monogene kognitive Störungen jedoch sehr hilfreich sein. Wie oben dargestellt, folgen zahlreiche überlappende Gene einem rezessiven Erbgang. Es ist denkbar, dass heterozygote Anlageträger eine IQ-Reduktion aufweisen, die eine wesentlich größere Effektstärke als assoziierte SNPs hat, was jedoch im polygenen Hintergrund untergeht. Auch hier hilft der Vergleich zum Merkmal Körperhöhe. Autosomal-rezessive Mutatio- nen im $A C A N$-Gen sind mit einer schweren Form des Kleinwuchses assoziiert. Heterozygote Anlageträger finden sich, wie erst kürzlich gefunden wurde, relativ häufig in Kohorten mit idiopathischem Kleinwuchs im Sinne eines Allels mit großer Effektstärke [17]. Auch Studien zu seltenen, nicht voll penetranten $\mathrm{Mi}$ krodeletionssyndromen können als Vorlage dienen. Bei der Mikrodeletion von Chromosom 16p11.2 zum Beispiel konnte der Vergleich mit Verwandten, die die Mikrodeletion nicht tragen, eine relative Reduktion von ca. 30 IQ-Punkten bei Deletionsträgern zeigen [54]. Je nach familiärem Hintergrund resultierte daher ein IQ noch im Norm- oder bereits im unterdurchschnittlichen Bereich.

Bei aller Schwierigkeit sind genetische Assoziationen meist wesentlich leichter und sicherer $\mathrm{zu}$ bestimmen, als das bisher für relevante Umweltfaktoren der Fall war. Diese tragen aber, wie oben dargestellt, ebenfalls signifikant zur Gesamtvarianz bei. Außerdem versprechen Umweltfaktoren, leichter modulierbar zu sein, weshalb gesicherte Erkenntnisse auch auf diesem Gebiet von großer Bedeutung sind. Studien zu Umweltfaktoren können zukünftig auch die genetische Komponente, z.B. über einen PGS, in den Analysen berücksichtigen, was ihre Power und Aussagekraft verbessern sollte. Auch die Erforschung der Gen-Umwelt-Interaktion wird nun mit der zunehmenden Aufklärung genetischer Grundlagen leichter möglich sein.

\section{Veränderbarkeit von Intelligenz}

Angesichts des großen öffentlichen Interesses am Merkmal Intelligenz und seiner Relevanz für die Erklärung und Vorhersage wichtiger Bereiche individuellen und gesellschaftlichen Erfolgs soll an dieser Stelle auf die Frage der Veränderbarkeit von Intelligenz eingegangen werden. Zunächst ist festzuhalten, dass klassische verhaltensgenetische Studien trotz der teils substanziellen Erblichkeitsbefunde grundsätzlich keine Aussage darüber erlauben, welche Veränderungspotenziale auf der Ebene des Individuums und in Bezug auf die absolute Ausprägung des Merkmals Intelligenz möglich sind, da die verwendeten Designs allein darauf abzielen, Einflüsse auf die bestehenden Merkmalsunterschiede zwischen Individuen zu quantifizieren. Gleichwohl sind die in diesem Beitrag berichteten Befunde an dieser Stelle von Relevanz.

Kinder aus höheren sozialen Schichten haben ungleich größere Chancen auf bessere Bildungsabschlüsse als Kinder aus niedrigeren sozialen Schichten. Vor dem Hintergrund der Tatsache, dass Intelligenz sich positiv auf den Bildungsund Lebenserfolg eines Menschen auswirkt und angesichts des Umstandes, dass Gene die Intelligenzentwicklung substanziell beeinflussen, lässt sich ableiten, dass der größere Bildungserfolg von Kindern aus höheren sozialen Schichten nicht allein mit einer besseren Förderung erklärt werden kann. Es kann davon ausgegangen werden, dass der Zusammenhang zwischen Bildungserfolg und Schichtzugehörigkeit teilweise genetische Ursachen hat. Bedingt ist dies möglicherweise durch Anlage-UmweltKorrelation (s. oben). Darüber hinaus gibt es aber auch Hinweise auf schichtspezifische Fördereffekte der Intelligenz, was $\mathrm{zu}$ dem bereits berichteten Befund passt, dass in bildungsferneren Familien geteilte Umwelteinflüsse bedeutsamer sind als in bildungsnahen Familien. Kinder aus bildungsfernen Schichten bleiben somit mit größerer Wahrscheinlichkeit hinter ihrem Potenzial zurück, es sei denn, sie werden durch geeignete Angebote gefördert. Es existieren empirische Hinweise auf die Veränderbarkeit von Intelligenz mittels Bildungsmaßnahmen [4] oder durch nachhaltige Veränderungen von Lebensbedingungen [11]. Eine systematische, vergleichende Betrachtung der Effektivität verschiedener aufwendiger Frühförderprogramme ist aufgrund der teils sehr unterschiedlichen Ziele und der verschiedenen Programmausgestaltung schwierig. Eine substanzielle und langfristig überdauernde Steigerung der Intelligenz scheint jedoch bisher nicht zu den positiven Effekten der Frühförderprogramme zu zählen [32] (vgl. dazu auch [38]).

Für Programme, die angeblich in unterschiedlichen Lebensphasen allgemeine mentale Funktionen trainieren können, fehlen überzeugende Nachweise ih- 
Hier steht eine Anzeige.

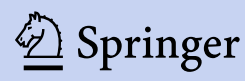


rer effizienten und nachhaltigen Wirksamkeit. Die in jüngerer Zeit medienwirksam propagierten Transfereffekte eines Trainings des Arbeitsgedächtnisses auf die Intelligenz halten einer kritischen Überprüfung nicht stand [28].

\section{Fazit für die Praxis}

Die jetzigen Befunde sind in erster Linie von wissenschaftlichem Interesse. Neben der weiteren Erforschung des Merkmals Intelligenz können sie evtl. auch Grundlage für oligogene Modelle sein, die insbesondere für das milde Spektrum der Intelligenzstörungen und Lernbehinderungen diskutiert werden. Darüber hinaus sind sie für die Erforschung von Umweltfaktoren von großer Bedeutung, die die Ausprägung des Merkmals beeinflussen können. Als Prädiktor für individuelle kognitive Fähigkeiten und Schulerfolg sind „polygenic scores" vor dem Hintergrund der probabilistischen Natur der Ergebnisse und der vergleichsweise geringen Varianzaufklärung im Kriterium ungeeignet.

\section{Korrespondenzadresse}

\section{Prof. Dr. med. André Reis}

Humangenetisches Institut, Universitätsklinikum Erlangen, Friedrich-Alexander-Universität Erlangen-Nürnberg (FAU)

Schwabachanlage 10, 91054 Erlangen,

Deutschland

andre.reis@uk-erlangen.de

Danksagung. Die Autoren danken Dr. Steffen Uebe, Erlangen, für den Abgleich mit der SysID-Datenbank und der Hilfe mit $\bullet$ Abb. 2.

\section{Einhaltung ethischer Richtlinien}

Interessenkonflikt. A. Reis und F.M. Spinath geben an, dass kein Interessenkonflikt besteht.

Dieser Beitrag beinhaltet keine von den Autoren durchgeführten Studien an Menschen oder Tieren.

Open Access. Dieser Artikel wird unter der Creative Commons Namensnennung 4.0 International Lizenz (http://creativecommons.org/licenses/by/4.0/deed. de) veröffentlicht, welche die Nutzung, Vervielfältigung, Bearbeitung, Verbreitung und Wiedergabe in jeglichem Medium und Format erlaubt, sofern Sie den/die ursprünglichen Autor(en) und die Quelle ordnungsgemäßnennen, einen Linkzur Creative Commons Lizenz beifügen und angeben, ob Änderungen vorgenommen wurden.

\section{Literatur}

1. Amthauer R, Brocke B, Liepmann D, Beauducel A (2001) Intelligenz-Struktur-Test 2000 R. Hogrefe, Göttingen

2. Bouchard TJ Jr., Mcgue M (1981) Familial studies of intelligence: a review. Science 212:1055-1059

3. Briley DA, Tucker-Drob EM (2013) Explaining the increasing heritability of cognitive ability across development: a meta-analysis of longitudinal twin and adoption studies. Psychol Sci 24:1704-1713

4. Brinch CN, Galloway TA (2012) Schooling in adolescence raises IQ scores. Proc Natl Acad Sci USA 109:425-430

5. Calvin CM, Batty GD, Der G et al (2017) Childhood intelligence in relation to major causes of death in 68 year follow-up: prospective population study. BMJ 357:j2708

6. Calvin CM, Deary IJ, Webbink D et al (2012) Multivariate genetic analyses of cognition and academic achievement from two population samples of 174,000 and 166,000 school children. Behav Genet 42:699-710

7. Carroll JB (1993) Human cognitive abilities:a survey of factor-analytic studies. Cambridge University Press, New York, NY

8. Davies G, Lam M, Harris SE et al (2018) Study of 300,486 individuals identifies 148 independent geneticloci influencing general cognitivefunction. Nat Commun 9:2098

9. Davies G, Marioni RE, Liewald DC et al (2016) Genome-wide association study of cognitive functions and educational attainment in UK Biobank ( $\mathrm{N}=112151)$. Mol Psychiatry 21:758-767

10. Deary IJ (2012) Intelligence. Annu Rev Psychol 63:453-482

11. Duyme M, Dumaret AC, Tomkiewicz S (1999) How can we boost IQs of "dull children"?: A late adoption study. Proc Natl Acad Sci USA 96:8790-8794

12. Ellinghaus D, Jostins L, Spain SLet al (2016) Analysis of five chronic inflammatory diseases identifies 27 new associations and highlights disease-specific patterns at shared loci. Nat Genet 48:510-518

13. Fromer $M$, Pocklington $A J$, Kavanagh $D H$ et al (2014) De novo mutations in schizophrenia implicate synaptic networks. Nature 506:179-184

14. Genetic Analysis of Psoriasis C, The Wellcome Trust Case Control C, Strange A, et al (2010) A genomewide association study identifies new psoriasis susceptibility loci and an interaction between HLACand ERAP1. Nat Genet 42:985-990

15. Gottfredson L (1997) Mainstream science on intelligence. An editorial with 52 signatories, history, and bibliography. Intelligence 24:13-23

16. Gow AJ, Johnson W, Pattie A et al (2011) Stability and change in intelligence from age 11 to ages 70 79, and 87: the Lothian Birth Cohorts of 1921 and 1936. Psychol Aging 26:232-240

17. Hauer NN, Sticht H, Boppudi S et al (2017) Genetic screening confirms heterozygous mutations in ACAN as a major cause of idiopathic short stature. SciRep 7:12225

18. Haworth CM, Davis OS, Plomin R (2013) Twins Early Development Study (TEDS): a genetically sensitive investigation of cognitive and behavioral development from childhood to young adulthood. Twin Res Hum Genet 16:117-125

19. Hill WD, Arslan RC, Xia C et al (2018) Genomic analysis of family data reveals additional genetic effects on intelligence and personality. Mol Psychiatry. https://doi.org/10.1038/s41380-0170005-1

20. Khera AV, Chaffin M, Aragam KG et al (2018) Genome-wide polygenic scores for common diseases identify individuals with risk equivalent to monogenic mutations. Nat Genet 50:1219-1224

21. Klauer KJ, Sparfeldt JR (2018) Intelligenz und Begabung. In: Rost DH, Sparfeldt JR, Buch SR(Hrsg) Handwörterbuch Pädagogische Psychologie, Bd. 5. PVU, Weinheim, S278-286

22. Kochinke K, Zweier C, Nijhof B et al (2016) Systematic Phenomics analysis Deconvolutes genes mutated in intellectual disability into biologically coherent modules. Am J Hum Genet 98:149-164

23. Koellinger PD, Harden KP (2018) Using nature to understand nurture. Science 359:386-387

24. Kong A, Thorleifsson G, Frigge ML et al (2018) The nature of nurture: effects of parental genotypes. Science 359:424-428

25. Lee JJ, Wedow R, Okbay A et al (2018) Gene discovery and polygenic prediction from a genomewide association study of educational attainment in 1.1 million individuals. Nat Genet 50:1112-1121

26. Macarthur J, Bowler E, Cerezo M et al (2017) The new NHGRI-EBI Catalog of published genomewide association studies (GWAS Catalog). Nucleic Acids Res 45:D896-D901

27. Marouli E, Graff M, Medina-Gomez C et al (2017) Rare and low-frequency coding variants alter human adult height. Nature 542:186-190

28. Melby-Lervag M, Hulme C (2013) Is working memory training effective? A meta-analytic review. Dev Psychol 49:270-291

29. Neisser U, Boodoo G, Bouchard TJ Jr et al (1996) Intelligence: knowns and unknowns. Am Psychol 51:77-101

30. Niemi MEK, Martin HC, RiceDLetal (2018)Common genetic variants contribute to risk of rare severe neurodevelopmental disorders. Nature. https:// doi.org/10.1038/s41586-018-0566-4

31. Okbay A, Beauchamp JP, Fontana MA et al (2016) Genome-wide association study identifies 74 loci associated with educational attainment. Nature 533:539-542

32. Olsen D (2005) Early childhood education: a caveat. Am ExpQ

33. Plomin R, Defries JC, Loehlin JC (1977) Genotypeenvironment interaction and correlation in the analysis of human behavior. Psychol Bull 84:309-322

34. Plomin R, Von Stumm S (2018) The new genetics of intelligence. Nat Rev Genet 19:148-159

35. Polderman TJ, Benyamin B, De Leeuw CA et al (2015) Meta-analysis of the heritability of human traits based on fifty years of twin studies. Nat Genet 47:702-709

36. Rietveld CA, Esko T, Davies G et al (2014) Common genetic variants associated with cognitive performance identified using the proxy-phenotype method. Proc Natl Acad Sci USA 111:13790-13794

37. Rietveld CA, Medland SE, Derringer J et al (2013) GWAS of 126,559 individuals identifies genetic variants associated with educational attainment. Science 340:1467-1471

38. Rost DH (2013) Handbuch Intelligenz. Beltz, Weinheim

39. Roth B, Becker N, Romeyke S, Schäfer S, DomnickF, Spinath FM (2015) Intelligence and school grades: a meta-analysis. Intelligence 53:118-137

40. Savage JE, Jansen PR, Stringer S et al (2018) Genome-wide association meta-analysis in 269,867 individuals identifies new genetic and functional links to intelligence. Nat Genet 50:912-919

41. Schmidt FL, Hunter J (2004) General mental ability in the world of work: occupational attainment and job performance. J Pers Soc Psychol 86:162-173 
42. Selzam S, Krapohl E, Von Stumm S et al (2017) Predicting educational achievement from DNA. Mol Psychiatry 22:267-272

43. Sniekers S, Stringer S, Watanabe Ket al (2017) Genome-wide association meta-analysis of 78,308 individuals identifies new loci and genes influencing human intelligence. Nat Genet 49:1107-1112

44. Snyderman M, Rothman S (1987) Survey of expert opinion on intelligence and aptitude testing. Am Psychol 42:137-144

45. Spinath FM, Klauer KJ (2018) Anlage und Umwelt. In: Rost DH, Sparfeldt JR, Buch SR (Hrsg) Handwörterbuch Pädagogische Psychologie, Bd. 5. PVU, Weinheim, S1-7

46. Stemmler G, Hagemann D, Amelang M, Spinath FM (2016) Differentielle Psychologie und Persönlichkeitsforschung Bd. 8. Kohlhammer, Stuttgart

47. Strenze T (2007) Intelligence and socioeconomic success: a meta-analytic review of longitudinal research. Intelligence 35:401-426

48. Trampush JW, Yang ML, Yu J et al (2017) GWAS meta-analysis reveals novel loci and genetic correlates for general cognitive function: a report from the COGENT consortium. Mol Psychiatry 22:336-345

49. Tucker-Drob EM, Bates TC (2016) Large crossnational differences in gene $x$ socioeconomic status interaction on intelligence. Psychol Sci 27:138-149

50. TurkheimerE, Beam CR, Sundet JM, Tambs K (2017) Interaction between parental education and twin correlations for cognitive ability in a Norwegian conscript sample. Behav Genet 47:507-515

51. Turkheimer E, Harden KP, D'onofrio B, Gottesman II (2009) The Scarr-Rowe interaction between measured socioeconomic status and the heritability of cognitive ability. In: McCartney K, Weinberg RA (Hrsg) Experience and development: a festschrift in honor of Sandra Wood Scarr. Psychology Press, New York, S81-97

52. Yengo L, Sidorenko J, Kemper KE et al (2018) Metaanalysis of genome-wide association studies for height and body mass index in approximately 700000 individuals of European ancestry. Hum Mol Genet. https://doi.org/10.1093/hmg/ddy271

53. Zahnleiter D, Uebe S, Ekici AB et al (2013) Rare copy number variants are a common cause of short stature. Plos Genet 9:e1003365

54. Zufferey F, Sherr EH, Beckmann ND et al (2012) A $600 \mathrm{~kb}$ deletion syndrome at $16 \mathrm{p} 11.2$ leads to energy imbalance and neuropsychiatric disorders. J Med Genet 49:660-668

\section{Vorläufer von Genen entstehen permanent "aus dem Nichts" - und verschwinden meist wieder}

\section{Bioinformatiker der Westfälischen Wilhelms-Universität Münster untersuchten erstmals die frühesten Stadien der Entstehung von "Genen aus dem Nichts" und publizieren die Arbeit in Nature Ecology and Evolution.}

Dass vollständige neue Gene und somit neue Eigenschaften quasi aus dem Nichts entstehen, galt jahrzehntelang als undenkbar. Erst Studien der jüngsten Zeit gaben vermehrt Hinweise darauf, dass sich neue Gene auch "aus dem Nichts" in der sogenannten nicht-codierenden DNA bilden, also in dem Teil des Erbguts, der keine Proteine erzeugt. Eine neue Arbeit untersucht nun erstmals auch die frühesten Stadien der Entstehung dieser "Gene aus dem Nichts". Die Arbeit, die aktuell in der Fachzeitschrift "Nature Ecology and Evolution" veröffentlicht ist, haben Bioinformatiker um Prof. Dr. Erich Bornberg-Bauer vom Institut für Evolution und Biodiversität der Westfälischen Wilhelms-Universität Münster (WWU) durchgeführt.

Das Team verglich mit Computeranalysen Anzahl, Länge, Position und Zusammensetzung (Nukleotid-Sequenz) von "Genen aus dem Nichts" beim Menschen mit denen von vier anderen Säugetier-Arten: Maus, Ratte, Kängururatte und Opossum. Letzteres gehört zu den Beuteltieren, deren Evolutionslinie sich früh von dem Zweig der Höheren Säugetiere abgespalteten hat. Durch diesen Vergleich warfen die Forscher Schlaglichter auf 160 Millionen Jahre Evolution der Säugetiere. Die Wissenschafter nahmen dabei DNA-Transkripte unter die Lupe, also solche DNA-Abschnitte, die aktiv sind und als RNA-Kopie vorliegen. Genauer gesagt untersuchten die Forscher die Transkripte sogenannter offener Leserahmen. Diese Sequenzen dienen häufig als Bauanleitungen für Proteine.

„Unsere Studie zeigt: Neue offene Leserahmen, also die Kandidaten für Bauanleitungen für neue Proteine, entstehen in nichtcodierenden DNA-Regionen permanent ,aus dem Nichts'. Sie gehen aber genauso wie ihre Transkripte im Laufe der Evolution auch sehr schnell wieder verloren", sagt Bioinformatiker Erich Bornberg-Bauer. Obwohl aus nur sehr wenigen dieser Kan- didaten tatsächlich funktionstüchtige Gene entstehen, also solche Gene, die den Bauplan für funktionierende Proteine enthalten, bleiben einige Kandidaten zufällig über längere Zeit erhalten - allein aufgrund der enormen Anzahl an ständig neu erzeugten Transkripten. „Diese Transkripte können dann in mehreren Abstammungslinien gefunden werden", so Erich BornbergBauer. „Wahrscheinlich können sie über lange Zeiträume hinweg das Repertoire der bestehenden Proteine ergänzen und an das molekulare Wechselspiel mit diesen angepasst werden."

Manchmal übernimmt also ein „aus dem Nichts" entstandenes Protein eine Funktion im Organismus. „Damit haben wir auch eine Erklärung dafür, wie grundlegend neue Eigenschaften in einem Organismus entstehen können. Allein durch punktuelle Veränderungen der genetischen Struktur ist das nämlich nicht erklärbar", so Erich Bornberg-Bauer.

Originalpublikation:

J.F. Schmitz, K.K. Ullrich, E. Bornberg-Bauer (2018): Incipient de novo genes can evolve from frozen accidents which escaped rapid transcript turnover. Nature Ecology and Evolution; DOI: 10.1038/s41559-018-0639-7

\section{Dr. C. Heimken, Presse- und Informationsstelle Westfälische Wilhelms-Universität Münster}

